\title{
In vitro gene silencing of independent phosphoglycerate mutase (iPGM) in the filarial parasite Brugia malayi
}

Prashant Kumar Singh, Susheela Kushwaha, Shahab Mohd, Manisha Pathak and Shailja Misra-Bhattacharya*

\begin{abstract}
Background: The phosphoglycerate mutase (PGM) enzyme catalyzes the interconversion of 2- and 3phosphoglycerate in the glycolytic /gluconeogenic pathways that are present in the majority of cellular organisms. They can be classified as cofactor-dependent PGM (dPGM) or cofactor-independent PGM (iPGM). Vertebrates, yeasts, and many bacteria have only dPGM, while higher plants, nematodes, archaea, and many other bacteria have only iPGM. A small number of bacteria, including Escherichia coli and certain archaea and protozoa, contain both forms. The silencing of ipgm in Caenorhabditis elegans (C. elegans) has demonstrated the importance of this enzyme in parasite viability and, therefore, its potential as an anthelmintic drug target. In this study, the role of the Brugia malayi (B. malayi) ipgm in parasite viability, microfilaria release, embryogenesis, and in vivo development of infective larvae post-gene silencing was explored by applying ribonucleic acid (RNA) interference studies.

Results: The in vitro ipgm gene silencing by small interfering RNA (siRNA) leads to severe phenotypic deformities in the intrauterine developmental stages of female worms with a drastic reduction ( $90 \%)$ in the motility of adult parasites and a significantly reduced (80\%) release of microfilariae $(\mathrm{mf})$ by female worms in vitro. Almost half of the in vitro-treated infective L3 displayed sluggish movement. The in vivo survival and development of siRNA-treated infective larvae ( $L 3$ ) was investigated in the peritoneal cavity of jirds where a $\sim 45 \%$ reduction in adult worm establishment was observed.
\end{abstract}

Conclusion: The findings clearly suggest that IPGM is essential for both larval and adult stages of B. malayi parasite and that it plays a pivotal role in female worm embryogenesis. The results thus validate the Bm-iPGM as a putative anti-filarial drug target.

Keywords: siRNA, Lymphatic filariasis, Drug target, RNAi, Embryogenesis

\section{Multilingual abstract}

Please see Additional file 1 for translations of the abstract into the six official working languages of the United Nations.

\section{Background}

Lymphatic filariasis (LF) is a vector-borne helminth disease caused by slender nematodes, Wuchereria bancrofti, Brugia malayi (B. malayi), and B. timori. This incapacitating disease infects over 120 million people in 72 tropical and subtropical countries, while more than

*Correspondence: shailja_cdri@rediffmail.com

Division of Parasitology, CSIR-Central Drug Research Institute, B.S. 10/1, Sector 10, Jankipuram Extension, Sitapur Road, Lucknow 226021, UP, India
1.39 billion people remain at the risk of infection [1]. The subclinical condition associated with patent infection may include acute manifestations, such as adenolymphangitis, acute dermatolymphangioadenitis, and tropical pulmonary eosinophilia that are rarely life threatening. However, chronic manifestations, such as lymphedema (elephantiasis) and hydrocele are debilitating, accounting for nearly five million disability-adjusted life years $[2,3]$. Current control measures for LF include annual doses of diethylcarbamazine or ivermectin alone, or in combination with albendazole, which principally targets microfilariae (mf) with little action on adult worms [4,5]. Target-based anthelmintic drug discovery is still at a nascent stage and only a few drug targets have been identified in filarial parasites utilizing this approach. The publication

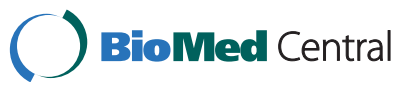


of the draft assembly of the B. malayi genome followed by an expansion in transcriptomic and genomic datasets has facilitated the identification of vital enzymes or proteins of filarial parasites that can be exploited as drug targets. This would further assist in the designing of potential antifilarial compounds and in understanding of gene functions $[6,7]$.

The gene silencing by ribonucleic acid (RNA) interference (RNAi) has been a landmark discovery in the area of biomedical research. RNAi has provided a functional genomics platform to unravel the functions of various genes and their associations with the disease state of the organism. 'Target validation' is an inherent aspect of the drug discovery process, which can be either accomplished by silencing the mRNA expression of a given gene in situ, or by using specific inhibitors against the target enzyme/protein to observe their deleterious effect on that organism. With a few exceptions, RNAi has not been utilized to its full extent in parasitic nematodes, including in filariids [8]. RNAi in Caenorhabditis elegans (C. elegans), a surrogate model for B. malayi, has been explored to identify the novel genes involved in nematode biology and comparative genomics studies have proposed a number of molecular targets for anti-parasitic drugs [9]. Another gene identified by RNAi in C. elegans is the 2, 3-bisphosphoglycerate independent phosphoglycerate mutase (iPGM). This enzyme's sequence and structure is completely different from the 2, 3-bisphosphoglycerate -dependent phosphoglycerate mutase (dPGM) found in mammals [10]. Both enzymes are responsible for the interconversion of 2-phosphoglycerate and 3-phosphoglycerate via different catalytic mechanisms. The down regulation of iPGM of C. elegans by RNAi resulted in embryonic and larval lethality [11]. Outcomes of RNAi experiments carried out on C. elegans do not always match those of parasitic helminths and, therefore, it becomes mandatory to carry out gene silencing in the target parasite itself [12]. The current investigation reports on the RNAi mediated ipgm gene silencing in adult and infective larval stages of human lymphatic filarial parasite, B. malayi. Using small size (small interfering) RNA (siRNA), the effect was observed on the parasite viability, the $\mathrm{mf}$ release, the adverse effect on the embryogenesis in the female worm, and further in vivo development of the treated infective larvae (L3) in the adult parasites in the peritoneal cavity of jirds.

\section{Methods}

\section{Animals}

Purpose-bred, parasite naïve, six- to eight-week old, male jirds (Meriones unguiculatus) were used in the study. Animals were maintained in proper housing conditions at an animal house facility at the Central Drug Research Institute (CDRI) in Lucknow, India, and fed a standard pellet diet and water ad libitum. The animals and the animal experimental procedures were approved by the Animal Ethics Committee of the Institute, duly constituted under the provisions of the Committee for the Purpose of Control and Supervision on Experiments on Animals (CPCSEA), Government of India. The study bears the approval no. 129/08/Para/IAEC/renew (84/09), dated 27.04.2009.

\section{Parasites and culture}

The $B$. malayi infective larvae (L3) were recovered from the laboratory bred vector mosquito, Aedes aegypti and fed $9 \pm 1$ days earlier on the donor Mastomys (multimammate mouse-Mastomys coucha) [13]. The L3 were isolated from the gently-crushed mosquitoes using the Baermann technique, washed repeatedly in the Ringer's solution, and counted. The six- to eight- week old male jirds were infected by inoculating 100-150 L3 into the peritoneal cavity of each animal. The adult parasites and the $\mathrm{mf}$ were recovered by washing the peritoneal cavity of the jirds after 120 to 180 days of intra-peritoneal infection. The $\mathrm{mf}$ were collected after passing the peritoneal lavage through a $5.0 \mu \mathrm{m}$ membrane filter (Whatman, USA). The parasites were washed repeatedly in the fresh culture medium RPMI 1640 (Sigma, USA) containing 100 units $/ \mathrm{ml}$ of penicillin and $100 \mu \mathrm{g} / \mathrm{ml}$ of streptomycin sulfate (Invitrogen, USA), and were transferred to an adequate volume of fresh medium, preheated to $37^{\circ} \mathrm{C}$ until they were ready to be used for RNAi experimentation. Undamaged, healthy, similar-sized, highly motile worms of both sexes, with females releasing almost near similar count of live $\mathrm{mf}$, were selected for the RNAi experimentation.

\section{Design and synthesis of siRNA}

The custom-designed and synthesized gene-specific siRNAs for Bm-iPGM used in this study were procured from Ambion, USA. The highest-ranking sense and antisense siRNA duplexes representing the best combination of activity and specificity were provided with a concentration of 40 nmoles as lyophilized powder. Stock solutions of $100 \mu \mathrm{M}$ were prepared and stored at $-20^{\circ} \mathrm{C}$ until use. The $5^{\prime}-3^{\prime}$ sequences of the sense and antisense strands of siRNA were:

\section{Sense (CCA UUG UGC UGA AAC AGA Att) Antisense (UUC UGU UUC AGC ACA AUG Gaa)}

The siRNA (\#AM 4621, Ambion) completely unrelated to $B$. malayi, that does not target any gene product, was used as a negative control to determine off-target effects, if any. The negative control siRNA did not have any sequence similarity to that of mouse, rat, or human gene sequences, and have been pretested (Ambion) in cell- 
based screens and proven to have no significant effect on cell proliferation, viability, or morphology.

\section{Demonstration of in vitro siRNA uptake by parasites using fluorescence microscopy}

The penetration of siRNA into B. malayi adult females, L3, and $\mathrm{mf}$ was observed after soaking the parasites in Cy3-labelled negative siRNA. Four female worms, 50 L3, and $100 \mathrm{mf}$ were soaked in the medium containing $2 \mu \mathrm{M}$ of siRNA separately for 24 hours at $37^{\circ} \mathrm{C}$. All three parasite stages were washed repeatedly in $\mathrm{PBS}(\mathrm{pH} 7.4)$, the fluorescence was visualized, and the parasites were photographed under a fluorescence microscope (Nikon, Japan) using a rhodamine filter set at an emission wavelength of $590 \mathrm{~nm}$.

\section{siRNA treatment of $B$. malayi adult worms by the soaking method}

The RNAi was carried out with the adult worms of both sexes by the soaking method. We used three groups in our experiments. The control group did not receive any siRNA treatment and the worms were kept in normal siRNA-free culture medium. The negative control group received the siRNA that was completely unrelated to $B$. malayi and the third experimental group received Bm-iPGM specific siRNA. The adult parasites (four female and two male worms) were taken in the midi GebaFlex tubes (cut off $5 \mathrm{kDa}$ ) containing $1 \mathrm{mM}$ spermidine, $8 \mathrm{U}$ RNaseOUT (Invitrogen, USA), $5 \mu \mathrm{M}$ of siRNA in $800 \mu \mathrm{l}$ of RPMI medium fortified with $10 \%$ fetal bovine serum (Invitrogen, USA) medium, and six such tubes were kept in a beaker containing $300 \mathrm{ml}$ of medium preheated to $37^{\circ} \mathrm{C}$. The beaker with the tubes was placed in a $\mathrm{CO}_{2}$ incubator at $37^{\circ} \mathrm{C}$ and the tubes were retracted at various time periods. The first tube was removed after 12 hours of incubation and the adult worms from the tube were transferred to a fresh siRNAfree medium. The leftover medium in the tube was centrifuged at $800 \times \mathrm{g}$ for two minutes, and the pellet was resuspended in another $50 \mu \mathrm{l}$ medium for further observation under the compound microscope to assess the number and phenotype of the in vitro released $\mathrm{mf}$. Of the four female worms, two were frozen in TRIzol reagent for later preparation of nucleic acid to measure mRNA expression of Bm-ipgm. The remaining two females and two males were transferred to the fresh preheated culture medium $\left(37^{\circ} \mathrm{C}\right)$ for 30 minutes, and their motility was assessed individually and scored. The viability of each worm was subsequently checked by MTT reduction assay using the dye 3-(4,5 dimethylthiazol2-yl)-2,5 diphenyltetrazolium bromide. The remaining three tubes were removed one by one after 24 hours, 36 hours and 48 hours, and were processed in the same way. The two leftover tubes were removed after 60 hours of incubation. Of the eight female worms obtained from these two tubes, two were frozen in the TRIzol reagent for their later use in PCR, while the remaining six females and four male worms were transferred to a fresh siRNA-free medium and were incubated for another 48 hours by replacing the medium with fresh normal medium every 24 hours. At the end of the experiment, i.e. 48 hours after shifting to the siRNA-free medium, two out of the six females were teased and their uterine contents were observed microscopically to determine the effect of gene silencing on embryogenesis by evaluating the relative proportion of various intrauterine progenies. The other two females were frozen in TRIzol reagent for PCR analysis, while the remaining two females and four male worms were checked for motility, viability, and in vitro $\mathrm{mf}$ release in the culture medium as discussed above. The experiment was repeated twice with the same number of worms under identical conditions and the data are expressed as the mean \pm SD of the two experiments.

\section{The effect of gene silencing on the in vitro release of $\mathrm{mf}$ and phenotypic changes}

The mf pellets, at various time points, were suspended in $50 \mu \mathrm{l}$ of the PBS as mentioned above, and $10 \mu \mathrm{l}$ of this suspension was used in triplicate for assessing the number of $\mathrm{mf}$ released in vitro. For observing the phenotypic changes, a thin smear of $\mathrm{mf}$ suspension was made on a glass slide, which was later fixed and stained with Giemsa and photographed (Nikon, Japan).

\section{The motility score and the viability of $B$. malayi adult worms}

The viability of the male and the female worms was assessed by the mitochondrial reduction of 3-[4,5dimethylthiazol-2-yl]-2,5-diphenyltetrazolium bromide (MTT, Sigma) to formazan, as described earlier [14]. The formation of the formazan was quantified spectrophotometrically at $530 \mathrm{~nm}$ in a multiplate reader (Tecan, Infinite M200, Switzerland), and the percent inhibition in MTT reduction by the treated parasite over that of control worms was assessed. The motility scoring of the adult worms was carried out as shown below and the percentage reduction in the motility of the worm over that of the control was evaluated. The worms displaying no movement even after transfer to the fresh siRNA-free medium were considered and scored as dead (see Table 1).

Table 1 Criterion for motility scoring of adult worms

\begin{tabular}{ccccccc}
\hline \multicolumn{7}{c}{ Motility of adult worms } \\
\hline \% Inhibition & 0 & 1 to 25 & 26 to 49 & 50 to 74 & 75 to 99 & 100 \\
\hline Score & 5 & 4 & 3 & 2 & 1 & Dead \\
\hline
\end{tabular}


Table 2 The PCR primers used for quantitative and RT-PCR analyses

\begin{tabular}{|c|c|c|}
\hline Target Gene & Primer name & Primers $\left(5^{\prime}-3^{\prime}\right)$ \\
\hline \multirow[t]{2}{*}{ Bm-ipgm } & Bm-ipgm F & $5^{\prime}$ GCT GAT AAG GTG ATT GAG 3' \\
\hline & Bm-ipgm R & $5^{\prime}$ CAG TTA CCA TCA GTA TGT AG 3 \\
\hline \multirow[t]{2}{*}{ Bm-tub-1 } & Bm-tub F & 5 ACT TGG TGT CCG AAT ATC3' \\
\hline & Bm-tub R & 5'ACTCTTCCTGTTCAATGTAT3' \\
\hline
\end{tabular}

\section{Measurement of the mRNA expression of Bm-ipgm}

The loss of specific transcripts following the RNAi treatment was examined by real-time quantitative RT-PCR (qRT-PCR). The B. malayi $\beta$-tubulin gene (Bm-tub-1) was used as an endogenous control gene. For qRT-PCR, the primers were designed with the Beacon designer software (see Table 2). The frozen worms were homogenized in the TRIzol reagent and the RNA was extracted as described earlier [15]. The first strand cDNA was generated using the Super Script III first strand cDNA synthesis kit (Invitrogen, USA) using oligo (dT) 20 primers. The specific cDNA fragments were amplified by the real-time PCR (Roche, USA) using a SYBR premix. The PCR conditions for qRT-PCR were $95^{\circ} \mathrm{C}$ for 5 minutes, followed by 40 cycles of $95^{\circ} \mathrm{C}$ for 20 seconds, $56^{\circ} \mathrm{C}$ for 15 seconds, and $72^{\circ} \mathrm{C}$ for 30 seconds. The relative amount of target amplicon in each experiment was determined by the comparative $\Delta C_{\mathrm{T}}$ method. The value of the control group (siRNA-free medium) was set to $100 \%$, and the relative reduction in RNAi treated groups was calculated and expressed as percentage reductions.

The silencing of the Bm-ipgm gene in infective $\mathrm{L} 3$

Two hundred L3 were kept in each of the three wells of a 48-well plate containing $1 \mathrm{ml}$ culture medium fortified with $1 \mathrm{mM}$ spermidine and $8 \mathrm{U}$ RNAse OUT. Of the three wells, one contained $2 \mu \mathrm{M}$ gene specific siRNAs, another one contained non-target siRNA, while the third one was devoid of siRNA. The plate was incubated at $37^{\circ} \mathrm{C}$ in $5 \% \mathrm{CO}_{2}$ for 24 to 48 hours, and the motility of the L3 was scored after 30 minutes of transfer to the fresh siRNA-free medium. The L3 that became totally immotile and did not display any sign of reversal in the motility after medium replenishment were considered to be dead. The L3 from individual wells were separately frozen in TRIzol reagent for analysis of Bm-iPGM transcript levels. Another set of experiments was conducted to observe the in vivo development of in vitro siRNA treated larvae in the naive jirds. The L3 were soaked in the gene-specific siRNA, non-target siRNA, and control media in the same manner as above for 24 hours, followed by washing in the siRNA-free medium, and 100 actively motile L3 each were subsequently inoculated in the peritoneal cavity of a 6 -week old male jird. A total of three jirds/ experiment could be inoculated with 100 L3 each as only active and live larvae were used for infecting jirds. The jirds were euthanized after 120 days post-infection and the parasites were isolated by repeated washing of the peritoneal cavity. The parasites were counted, measured, and females were later teased individually in a drop of PBS to observe the intrauterine development.

\section{Statistical analysis}

The data were analyzed using one-way and two-way (for grouped data) analysis of variance (ANOVA) with the help of statistical software PRISM 5.0. The individual comparisons following ANOVA were made using the Bonferroni method or the Newman-Keuls Multiple Comparison Test, wherever applicable. The criterion of evaluating statistical significance between the experimental and control groups was as follows: $p$ value $<0.05$ was considered significant and marked as *, $p<0.01$ as highly significant and marked as $\%$, and $p<0.001$ was very highly significant and marked as $\% *$.

\section{Results}

The soaking method successfully delivered the siRNA

All three parasite stages were incubated in vitro in the presence of $2 \mu \mathrm{M}$ concentration of Cy3-labeled negative control siRNA to ensure the proper uptake of siRNA by

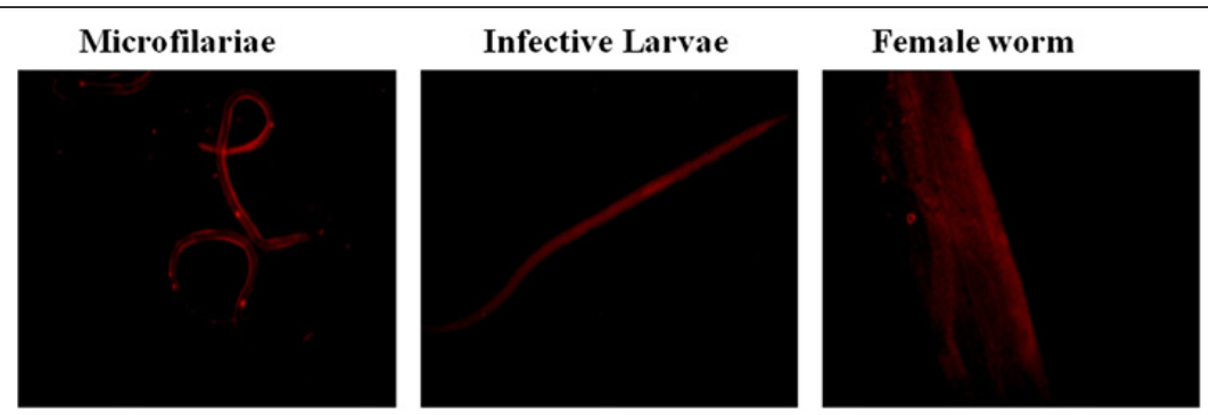

Figure 1 Confirmation of penetration of siRNA to $\mathbf{m f}, \mathbf{L 3}$, and adult female worms. The Cy3-labeled negative control siRNA was taken up by all three stages of parasite in vitro after soaking for 24 hours at $2 \mu \mathrm{M}$ concentration. The fluorescence was visualized using a Nikon fluorescence microscope using the rhodamine filter set at emission $590 \mathrm{~nm}$. 


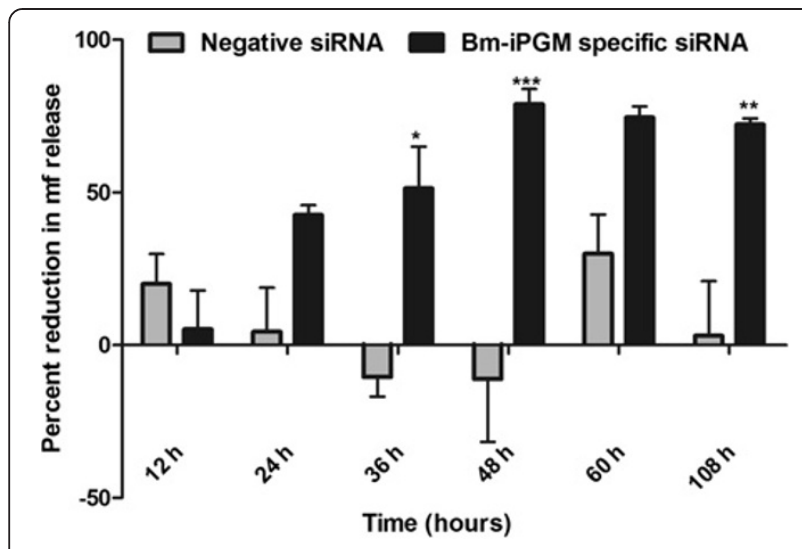

Figure $\mathbf{2}$ The in vitro siRNA treatment of adult female B. malayi leads to a reduction in microfilaria release. Tubes containing six adult worms each were treated with $5 \mu \mathrm{M}$ siRNA for different time periods $(12,24,36,48,60$ hours). After 60 hours of treatment, six females were cultured for a further two days in fresh siRNA-free normal culture medium (108 hours). The percent reduction in the $\mathrm{mf}$ release was analyzed in the Bm-iPGM-specific siRNA-treated and negative control group over that of the control media group. There was a significant reduction in the release of $\mathrm{mf}$ by Bm-iPGM siRNAtreated worms starting from 12 hours and continuing until 108 hours. The criterion for statistical significance between the results was carried out by ANNOVA and was expressed in the form of $p$ value that was considered significant and marked as $p<0.05 *$, $p<0.01$ as highly significant ${ }^{* *}$, and $p<0.0011^{* * *}$ as very highly significant. Data represent mean $\pm \mathrm{SE}$ of percent reduction in $\mathrm{mf}$ counts from two independent experiments.

the soaking method. The siRNA uptake occurred successfully in all three stages of B. malayi at 24 hours of soaking (see Figure 1). Fluorescence could be clearly detected at 24 hours in the hypodermis, intestine, and uterus of the female worms, while in the L3 and mf, siRNA could be visualized throughout the body of the filarial parasites.

The silencing of the B. malayi ipgm gene impairs the adult $B$. malayi viability

The siRNA-mediated silencing of the Bm-ipgm gene by the soaking method resulted in the reduced motility of both male and female worms as compared to that of the control worms, which were exposed either to the normal, medium, or negative siRNA (see Table 3). Male worms were more sluggish in appearance as compared to the female worms in the experimental groups. The siRNA-treated parasites showed reduced viability in MTT reduction tests, however, both the male and female worms remained alive until the end of the experiment (60 hours), and also after 48 hours of transfer to the siRNA-free medium (total 108 hours). The treated worms demonstrated a continuous decrease in their motility with the increasing duration of incubation in specific siRNA, and the motility data correlated well with the inhibition in MTT reduction. Maximum inhibitions in MTT reductions in treated female and male worms were observed to be $75.9 \%$ and $89.4 \%$, respectively. After 36 hours of soaking in the medium-containing target siRNA, both sexes of adult parasites demonstrated $\geq 50 \%$ inhibition in MTT reduction that exhibits a significant adverse effect on parasite viability [16].

The Bm-ipgm silencing impairs the release of $\mathrm{mf}$ by the female worms, mf motility and their phenotype in vitro The $\mathrm{mf}$ released by the controls and the siRNA-treated female B. malayi in culture medium were counted. A considerable drop was noticed in the number of $\mathrm{mf}$ released by the Bm-ipgm specific siRNA-treated female parasites within 24 hours of incubation, which decreased further up to $\sim 80 \%(p<0.001)$ at 48 hours, indicating a potent adverse effect of gene silencing on $\mathrm{mf}$ release. This effect remained unchanged even after transferring these worms to a siRNA-free medium. No such pattern was observed in the non-specific siRNA-treated control worms (see Figure 2). In addition, a complete loss of motility was also witnessed in $\sim 80 \%$ of the released $\mathrm{mf}$ (these appeared dead) within 24 hours of exposure and partial paralysis was observed in the remaining $20 \% \mathrm{mf}$ (these seldom even had slight movement of the anterior or posterior portion tips). The phenotypic deformities in the $\mathrm{mf}$ were conspicuous by contraction of the body at one end, leaving most of the mf sheath empty. Besides this, a few small and large vacuoles were also visible inside the non-motile $\mathrm{mf}$ in the Bm-ipgm specific siRNAtreated group (see Figure 3).

Table 3 Effect of Bm-ipgm gene silencing on adult male and female B. malayi

\begin{tabular}{|c|c|c|c|c|c|c|c|c|c|c|}
\hline \multirow[b]{2}{*}{ Time (hr) } & \multicolumn{3}{|c|}{$\begin{array}{c}\text { Motility score } \\
\text { (female worms) }\end{array}$} & \multicolumn{2}{|c|}{$\begin{array}{c}\% \text { Inhibition in MTT } \\
\text { reduction }\end{array}$} & \multicolumn{3}{|c|}{$\begin{array}{c}\text { Motility score } \\
{ }^{*}(\text { male worms) }\end{array}$} & \multicolumn{2}{|c|}{$\begin{array}{c}\% \text { Inhibition in MTT } \\
\text { reduction }\end{array}$} \\
\hline & Control & $\begin{array}{l}\text { Negative } \\
\text { siRNA }\end{array}$ & $\begin{array}{l}\text { Gene specific } \\
\text { siRNA }\end{array}$ & $\begin{array}{l}\text { Negative } \\
\text { SiRNA }\end{array}$ & $\begin{array}{l}\text { Gene specific } \\
\text { siRNA }\end{array}$ & Control & $\begin{array}{l}\text { Negative } \\
\text { siRNA }\end{array}$ & $\begin{array}{c}\text { Gene specific } \\
\text { SiRNA }\end{array}$ & $\begin{array}{l}\text { Negative } \\
\text { siRNA }\end{array}$ & $\begin{array}{l}\text { Gene specific } \\
\text { siRNA }\end{array}$ \\
\hline 12 & 5 & 5 & 4 & $2.25 \pm 3.3$ & $29.9 \pm 5.4$ & 5 & 5 & 4 & $2.5 \pm 1.3$ & $26.8 \pm 4.5$ \\
\hline 24 & 5 & 5 & 3 & $4.5 \pm 1.2$ & $40.2 \pm 6.2$ & 5 & 5 & 3 & $4.5 \pm 1.3$ & $38.8 \pm 6.5$ \\
\hline 36 & 4 & 5 & 3 & $5.0 \pm 2.3$ & $55.4 \pm 6.3$ & 4 & 4 & 2 & $4.5 \pm 2.6$ & $64.4 \pm 5.4$ \\
\hline 48 & 4 & 4 & 2 & $6.2 \pm 2.8$ & $74.4 \pm 3.4$ & 4 & 4 & 1 & $6 \pm 1.8$ & $80.2 \pm 6.8$ \\
\hline 108 & 4 & 4 & 2 & $6.26 \pm 2.3$ & $75.9 \pm 4.7$ & 4 & 4 & 1 & $5.25 \pm 2.6$ & $89.4 \pm 5.3$ \\
\hline
\end{tabular}

Key: ${ }^{*} 0 \%: 5 ; 1$ to $25 \%: 4 ; 26$ to $49 \%: 3 ; 50$ to $74 \%: 2 ; 75-99 \%: 1 ; 100 \%$ : Dead. 
The Bm-ipgm gene silencing had profound adverse effects on female worm embryogenesis

At the end of the experiment (108 hours after initiation of RNAi treatment), the female worms were collected for preparing the embryogram (see Figure 4). The relative proportion of various progenies at different stages of development that displayed good proportions of the intrauterine contents in the form of degenerated eggs
( $40 \%)$ was noted, and the complete morphogenesis seemed to have failed. The remaining stages consisted of eggs, early embryos, pretzel and, full-grown $\mathrm{mf}$. The silencing of the Bm-ipgm gene caused the abnormality in the phenotypes of the eggs and the mf. All the developing stages appeared normal in the culture medium devoid of the target siRNA, or the non-target probe (see Figure 3).
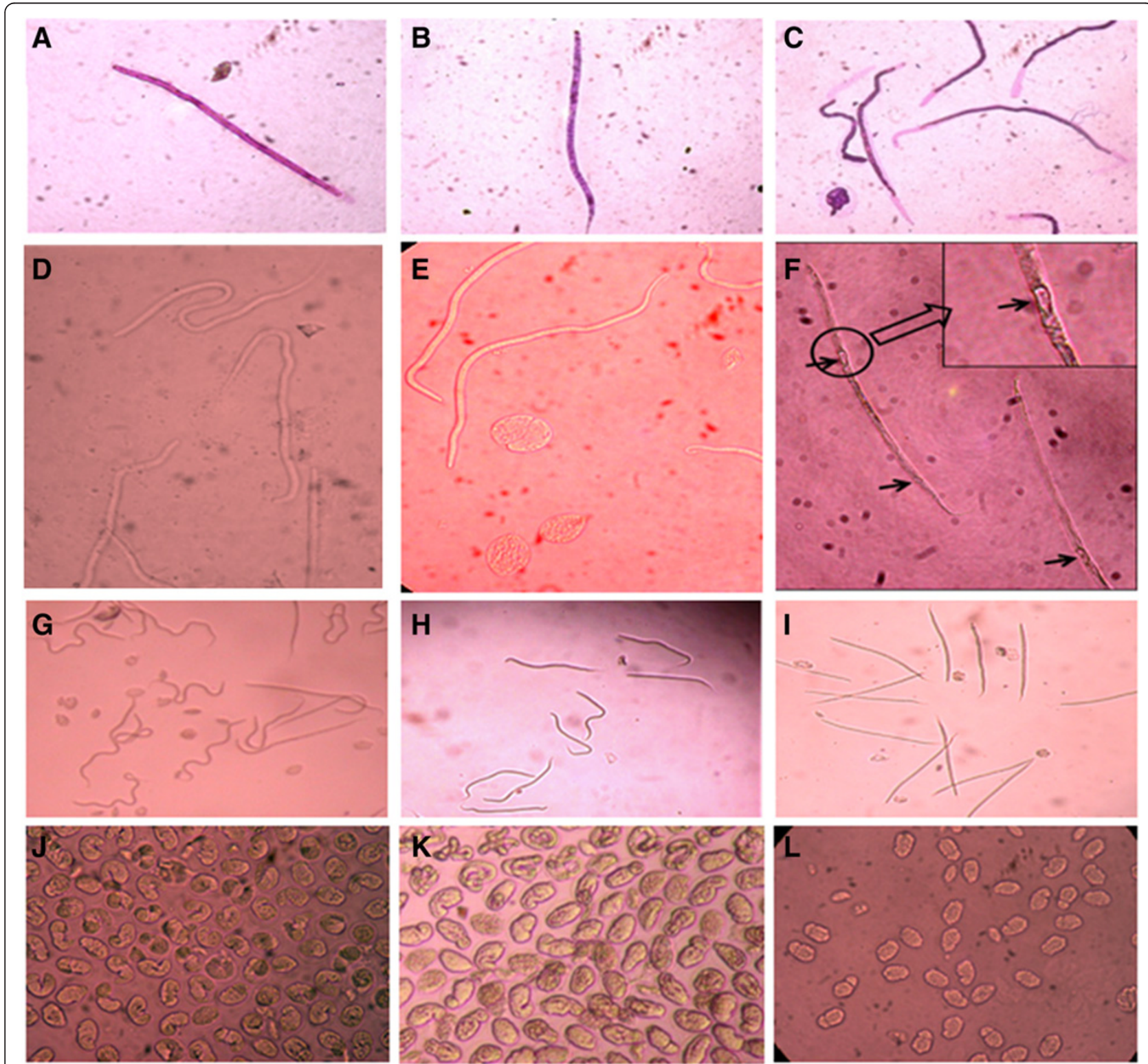

\section{Medium control}

Non specific siRNA control

Bm-iPGM specific SiRNA

Figure 3 The Bm-ipgm specific gene silencing causes several developmental defects in the $\mathrm{mf}$ and the eggs. The Giemsa-stained $\mathrm{mf}$ from the control group (A), siRNA-free negative control group (B), and the Bm-iPGM specific siRNA-treated group (C) exhibited stretched mf sheaths. The $\mathrm{mf}$ from the control groups ( $\mathbf{D}$ and $\mathbf{E}$ ) had normal phenotypes as compared to Bm-iPGM-specific siRNA treatment that led to variable phenotypes in the $\mathrm{mf}$ including degenerating internal contents containing several vacuoles ( $\mathbf{F}$, arrows). The $\mathrm{mf}$ from the control groups were alive $(\mathbf{G}, \mathbf{H})$, while those from the specific siRNA-treated group were dead (I). The eggs from the control groups $(\mathbf{J}, \mathbf{K})$ were normal, while those from the iPGM specific siRNA (L) were degenerated, showing increased spaces between the eggshell and the embryo. 


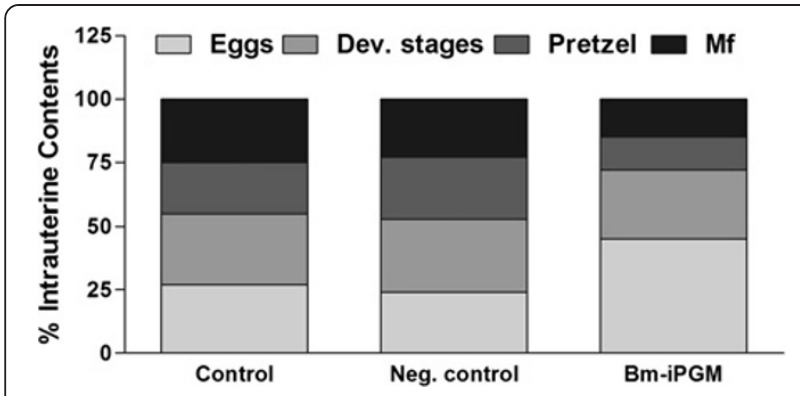

Figure 4 The effect of siRNA on B. malayi female worm embryogenesis. The embryograms of the female worms exposed to the culture medium, the negative siRNA, and the Bm-iPGM -specific siRNA $(5 \mu \mathrm{M})$ were examined after 108 hours of incubation. The data are expressed as percentages of eggs, early developing stages, pretzel, and $\mathrm{mf}$.

Soaking of the adult B. malayi in the Bm-ipgm siRNAcontaining medium leads to the loss of the Bm-iPGM transcript

The qRT-PCR results showed that the Bm-ipgm gene silencing resulted in the transcript reduction relative to the expression of the $\beta$-tubulin control gene within 12 hours, which continued further until 60 hours. This reduction in the gene expression profile of the siRNAsoaked worms was irreversible even after 48 hours of transfer to the siRNA-free medium (see Figure 5). The control worms revealed the presence of the Bm-iPGM transcript at all time points of the study. The reduction in the Bm-iPGM specific transcript level was highly significant $(p<0.001)$ when compared with the negative control, which had negligible (5-8\%) reduction. The data have been represented as the percentage reduction in the gene expression normalized using $\beta$-tubulin (Bmtub-1) transcript as the housekeeping gene control. The reduction in the transcript levels correlated well with the reduction in the release of $\mathrm{mf}$ from the target specific siRNA-treated female worms, phenotypic abnormalities, and with the complete loss of motility of the released $\mathrm{mf}$, as well as with adverse effects on the intrauterine development.

The Bm-ipgm gene silencing in the infective $L 3$ reduces their motility and impairs their further development in jirds In vitro silencing of the Bm-ipgm gene in the L3 brought about a reduction in the motility of almost half the cultured L3 at 48 hours in contrast to the control group, where $10-12 \%$ of the L3 became sluggish. Under normal culture conditions in the normal medium, around 25\% of the L3 became non-motile and were considered dead. A similar effect was also observed in the target siRNAtreated group (see Table 4) demonstrating no noticeable adverse effects of siRNA on larval viability. However, the gene silencing had profound adverse effects on the L3 motility leading to sluggishness but not amounting to

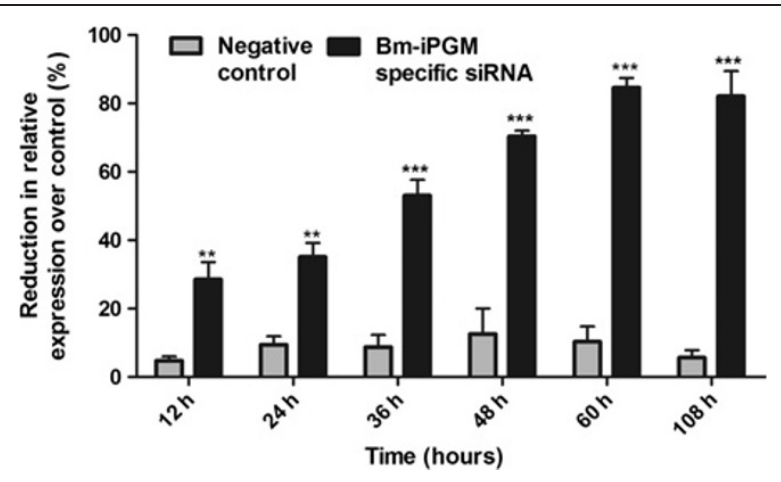

Figure 5 The percent reduction in gene transcript level in $\mathrm{Bm}$ ipgm specific siRNA treated worms. Two female worms at different time intervals $(12,24,36,48,60$ hours) and 48 hours after transfer to normal siRNA-free culture medium were removed and analyzed for differences in Bm-ipgm gene-specific transcript levels by qRT-PCR. The relative amounts of iPGM amplicon were determined by using the comparative $\Delta C T$ method and normalizing against the endogenous control gene (Bm-tub-1). The median value of the control group was set to $100 \%$ and the reduction in expression in the treated groups was calculated as a percentage of the control. The criterion for statistical significance between the results was carried out by ANNOVA and was expressed in the form of $p$ value that was considered significant and marked as $p<0.05^{*}, p<0.01$ as highly significant ${ }^{* *}$, and $p<0.001{ }^{* * *}$ as very highly significant. Data represent mean \pm SE of percent reduction in $\mathrm{mf}$ counts from two independent experiments.

death within 48 hours of observation. There was $~ 65 \%$ reduction in the $\mathrm{Bm}$-iPGM transcript level as revealed by the qRT-PCR of these L3 at 24 and 48 hours of observation (see Figure 6A). The surviving active and motile L3 from all three groups from the different batches were separately inoculated into the peritoneal cavity of the naïve jirds to observe further in vivo development of these L3s. There was a $44.36 \%$ reduced recovery rate $(p<0.01)$ of adult parasites when jirds were euthanized on day 120, in comparison to those recovered from control jirds (see Figure 6B). A proportion of recovered female worms $(24.9 \pm 6.8 \%)$ from experimental jirds contained degenerated intrauterine contents (see Figure 6C). An interesting observation was that the relative establishment of female worms was very low in the jirds infected with the target gene specific siRNA-treated L3 in contrast to the female worm recovery from the two control groups. The sizes of the adult parasites were also marginally shorter than those isolated from the control jirds (see Table 5).

\section{Discussion}

Phosphoglycerate mutases are the enzymes that catalyze the interconversion of 2- and 3-phosphoglycerate in the glycolytic/gluconeogenic pathways that are present in most cellular organisms. High-throughput RNAi studies performed in $C$. elegans have identified several genes essential for parasite viability including the cofactor- 
Table 4 The effect of Bm-iPGM siRNA treatment on the morphology of infective larvae (L3)

\begin{tabular}{|c|c|c|c|c|c|c|}
\hline \multirow{2}{*}{$\begin{array}{l}\text { Infective Larvae } \\
\text { condition }\end{array}$} & \multicolumn{2}{|c|}{ Control } & \multicolumn{2}{|c|}{ Negative control } & \multicolumn{2}{|c|}{ Bm-iPGM specific siRNA } \\
\hline & $24 \mathrm{~h}$ & $48 \mathrm{~h}$ & $24 \mathrm{~h}$ & $48 \mathrm{~h}$ & $24 \mathrm{~h}$ & $48 \mathrm{~h}$ \\
\hline Active (\%) & $82.5 \pm 4.9$ & $58.5 \pm 12.0$ & $82.5 \pm 7.7$ & $57.5 \pm 9.1$ & $49 \pm 2.12$ & $23.5 \pm 9.1$ \\
\hline Sluggish (\%) & $10.0 \pm 2.8$ & $17.5 \pm 3.5$ & $12.5 \pm 3.5$ & $17.0 \pm 5.6$ & $37.0 \pm 2.12$ & $50.5 \pm 3.5$ \\
\hline Dead* (\%) & $7.5 \pm 2.2$ & $24.0 \pm 8.4$ & $10.0 \pm 2.8$ & $25.5 \pm 3.5$ & $14.0 \pm 4.24$ & $26.0 \pm 12.7$ \\
\hline
\end{tabular}

*: These were the non-motile L3 where no reversal in motility was noticed even after transfer to siRNA-free medium.

independent phosphoglycerate mutase (iPGM) [17-20]. The iPGM is present in almost all nematodes, bacteria, and trypanosomes with the exception of some bacteria, such as E. coli, and certain archaea and protozoa that contain both the iPGM and APGM forms. [21-27]. Because of its absence from the vertebrates and its key role in pathogen development, this enzyme shows great potential as a possible drug/vaccine candidate. In this study, attempts were made to investigate the functional role of the B. malayi ipgm gene in parasite viability, $\mathrm{mf}$ release, embryogenesis, and in vivo infective L3 development by RNAi mediated silencing using two important life stages of $B$. malayi, the L3 and adult worms.

The RNAi was discovered accidentally in C. elegans $[28,29]$ and has been tried with parasitic nematodes with variable and limited success. Nippostrongylus brasiliensis was the first nematode for which RNAi effects were reported using double-stranded RNAs (dsRNAs) [30] and since then, RNAi has been reported from various other nematodes, such as Haemonchus contortus [31-33], Ascaris suum [34-36], Trichostrongylus colubriformis [35,37], O. volvulus [38,39], Ostertagia ostertagi [37], and B. malayi [40]. Recently, in vivo silencing has also been demonstrated in Aedes aegypti, the mosquito vector of $B$. malayi [41]. We have recently reported on the use of 19 bp siRNA to successfully silence $B$. malayi RNA helicase by both soaking and electroporation methods [42].

Selection of siRNAs appropriate for a given sequence are of prime importance and are mainly based on structural attributes, such as optimal length, concentration, the requirement of a 3' dinucleotide overhang, and a low G/C content [43-46]. Initially, we used 21 bp siRNA for soaking the mf, L3, or adult worms at $2 \mu \mathrm{M}$ concentration of Cy3-labeled, non-specific siRNA, which showed a successful penetration of siRNA in all three parasite stages. However, $5 \mu \mathrm{M}$ of siRNA was used for soaking adult parasites in our experiments, as this concentration was effective enough to bring down the silencing effects in our previous studies. For the gene silencing in the L3, a lower concentration $(2 \mu \mathrm{M})$ of siRNA was used as L3s demonstrated better absorption of siRNA at this lower concentration. The low concentrations and small-sized dsRNA/siRNA have also been found to produce more effective gene silencing effects as opposed to high
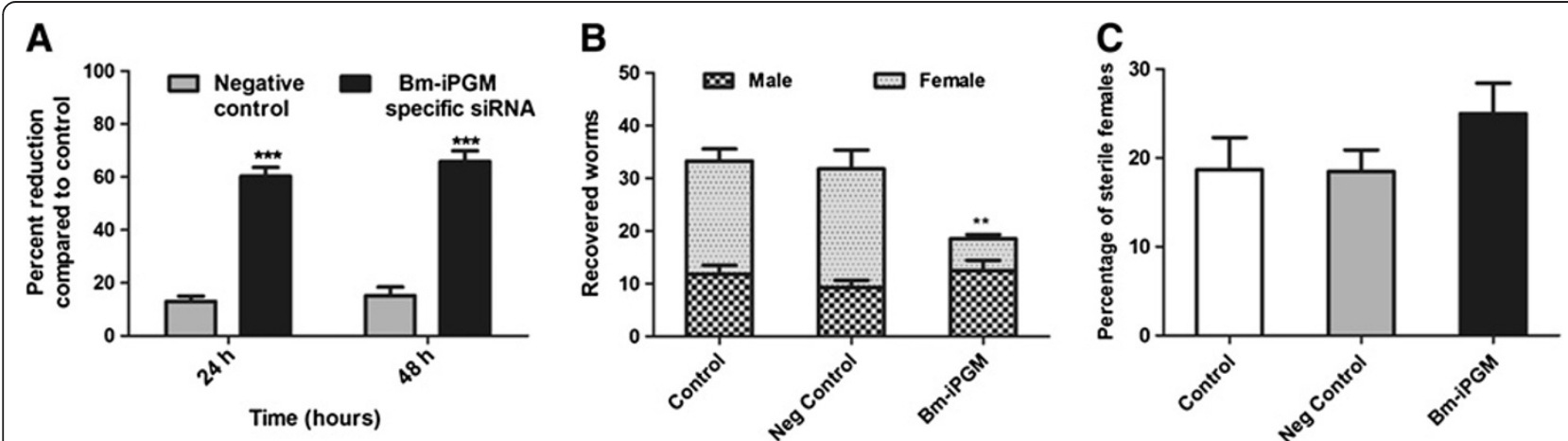

Figure 6 Effect of siRNA on Bm-ipgm gene transcription in the L3 stage and the production of adult worms. (A) siRNA treatment of infective larvae ( $L 3$ ) resulted in a significant loss of the Bm-iPGM transcript level after 24 and 48 hours of incubation. (B) The number of recovered male and female worms from the control, negative control, and Bm-ipgm-specific treated L3 implanted jirds. The Bm-ipgm-specific siRNA-treated L3 exhibited $44.36 \%$ reduction in the adult worm establishment in the peritoneal cavity of naive male jird, which was significant $(p<0.01)$ over control. (C) The percentage of sterile female worms recovered from control, negative control, and Bm-ipgm-specific treated L3 implanted jirds. A significant number of female worms recovered from the siRNA-treated group displayed defective embryogenesis. The recovered females were teased in a drop of PBS on glass slide and the condition of intrauterine contents was analyzed microscopically (Nikon). The percentage of sterile female worms was calculated with respect to the total female worms recovered and analyzed. The criterion for statistical significance between the results was carried out by ANNOVA and was expressed in the form of $p$ value that was considered significant and marked as $p<0.05 *$, $p<0.01$ as highly significant ${ }^{* *}$, and $p<0.001{ }^{* * *}$ as very highly significant. The data represent the mean $\pm \mathrm{SE}$ of percent reduction in mf counts from the two independent experiments. 
Table 5 Measurement of worm length recovered from jirds infected with siRNA-treated and untreated infective larvae

\begin{tabular}{lll}
\hline Groups & \multicolumn{2}{c}{ Worm length $(\mathbf{c m} \pm$ S.D.) } \\
\cline { 2 - 3 } & Female & Male \\
\hline Control & $3.47 \pm 0.57$ & $1.67 \pm 0.34$ \\
\hline Negative control & $3.48 \pm 0.65$ & $1.75 \pm 0.25$ \\
\hline Bm-iPGM specific siRNA & $2.60 \pm 0.70$ & $1.58 \pm 0.29$ \\
\hline
\end{tabular}

concentrations that induce stress in the parasites. The small-sized siRNA reduces the innate immune interferon response and produces negligible non-target effects [42,47-49]. The single siRNA was computationally designed and custom synthesized by Ambion to target Bm-iPGM.

Several studies have reported on off-target effects of gene silencing and, therefore, use of more than one siRNA has been recommended to confer effective gene silencing. To confirm that the observed effects were gene specific, a negative control completely unrelated to B. malayi was used in this study. However, the use of another siRNA specific to Bm-iPGM would have given our results even more credibility. In previous studies, we have used a single siRNA to target specific genes of interest and were substantially successful in gene silencing without observing any off-target effects $[38,46]$. The soaking method was used, as described earlier $[40,42,50]$, with substantial modifications. Worms were kept in different tubes so that each tube could be taken out at any time point without touching the remaining worms in the other tubes. This was done to avoid any mechanical damage, stress, or contamination to the remaining parasites requiring longer incubation. The loss of gene transcript level started within 12 hours of soaking in the Bm-iPGM specific siRNA, and was apparent until the end of the experiment, not showing any reversal even after 48 hours of further transfer of worms to the siRNA-free medium. The loss of gene function slowed down the motility of both the male and female parasites. The percent inhibition in parasite motility correlated very well with the MTT assay, which is a quantitative assay and has been widely used for evaluation of in vitro anti-filarial activity of compounds against $B$. malayi worms. There was a specific knockdown of the BmiPGM transcript along with a range of deleterious effects on the phenotypes, however, additional studies could not be performed to detect the degree of protein knockdown. This was due to the limited number of experimental and control worms, and these were utilized for assessing other parameters thought to be more important for the study.

The measurement of the $\mathrm{mf}$ release in the culture is a robust method to ensure the ill effects of gene silencing on the female parasites. The profound decrease in the number of $\mathrm{mf}$ released by the specific siRNA-treated female worms indicated the deleterious effects on worm function. The released $\mathrm{mf}$ revealed phenotypic deformities in the form of a contracted body at one end, leaving a long sheath at the other end, as well as internal vacuole formation with subsequent loss of motility, amounting to death. The relative proportion of various progenies demonstrated failure of complete morphogenesis. The number of free $\mathrm{mf}$ and pretzel stages within the uteri of the female worms showed considerable reduction with increased proportion of early egg stages showing impairment in the transformation of the divided eggs into the pretzel stages. This might have been brought about by the insufficient production of energy needed for germ-cell differentiation since iPGM is a crucial enzyme of the parasite glycolytic pathway. It has been shown that mitochondrial content is regulated during transition from different parasitic stages and different stages have different energy requirements [51]. iPGM is involved in fundamental metabolic pathway and has been shown in C. elegans to be expressed throughout the worm in all the developmental stages, being abundant in the cells with higher metabolic rates, such as contracting body wall muscles, nerve ring, and the intestinal cells.

The disruption of the C. elegans iPGM by RNAi brought about variable defects, including embryonic and larval lethality [11]. Almost half of the in vitro siRNAtreated L3 became sluggish, while a further 25\% remained totally immotile even after transfer to normal culture media and were thus considered 'dead'. Even though, the gene silencing effects were conspicuous on the motility and viability of L3, it could not lead to their death. The possible reason for infective L3 survival could be the abundant storage of the iPGM enzyme in the worm body, or the slow and sustained depletion of the iPGM transcript of up to $90 \%$, but not $100 \%$. A single concentration of siRNA was used in the current study due to its high cost, however, increasing the duration of the siRNA treatment or the use of different concentrations of siRNA may have possibly shown dose dependent effects on $B$. malayi.

A successful RNAi experiment depends on various factors, including culture conditions, route/mode of siRNA delivery, the gene targeted, its site, the level of expression in the parasite, and the parasite itself. This indicates differences between nematodes in the uptake and the spread of dsRNA/siRNA that could have been the reason for the variation in the results obtained with different parasites. Silencing of the iPGM in C. elegans had some differing outcomes that could be because of the differences in the RNAi methodology used by us and Zhang et al., 2004 [10]. The latter have used dsRNA instead of siRNA, which was used in our study. The 
dsRNA/siRNA design could inform variable efficiencies of different dsRNAs/siRNAs or individual transcript sensitivities within the individual species, and this could have been one of the reasons for the differences observed. However, in C. elegans, the embryonic lethality started within 18 to 24 hours, reaching a maximum of $94 \%$ at 50 to 60 hours post injection of the dsRNA.

The in vivo survival and the development of siRNAtreated L3 was further investigated by peritoneal inoculation in the naive jirds and euthanizing of the animals on day 120 to observe the establishment of these larvae into the adult parasites. The in vivo results demonstrated the inability of a good proportion of the treated infective L3s to reach adult stage. Not only this, but the established adult worms had profound retardation in their lengths over those who recovered from the control groups. In addition, there was an unusually low recovery rate of female worms, which was less than half of the recovered males. This may be due to differential $\mathrm{Bm}$ ipgm expression patterns in male and female worms. In one study, it has been shown that approximately $4 \%$ of genes are associated with glycolysis and gluconeogenesis in male worms as compared to only $1 \%$ in female worms [52]. Thus, silencing of the ipgm might have had a more fatal effect on the carbohydrate metabolism in female worms resulting in their reduced recovery. The control groups had almost twice the number of female worms than male worms, which is quite usual in B. malayiinfected animals. The recovered female worms from the experimental group also displayed defective embryogenesis. These in vivo studies confirm the role of iPGM in nematode metabolism, growth, and fecundity. The adverse effects of iPGM gene silencing on growth have been demonstrated earlier in a variety of plants and human parasites. The double iPGM mutants in Arabidopsis thaliana and iPGM antisense potato plants showed severely-retarded plant growth that correlated with the decreased concentrations of phosphoenolpyruvate $[53,54]$. The iPGM is required for the normal growth of Trypanosoma brucei procyclic [26] and the blood stream form [55], and has been proposed as a drug target in spore-forming Bacillus species and tomato pathogen, Pseudomonas syringae $[56,57]$.

The present findings clearly demonstrate the vital role of iPGM, a metabolic enzyme in parasite growth, and validates Bm-iPGM as a possible anti-filarial drug target.

\section{Conclusion}

The identification and validation of anti-filarial drug target/s is of extreme importance as there is currently no adulticidal anti-filarial drug. RNAi has been widely used to validate and identify such drug targets, and PGM is one such enzyme found in two forms: vertebrates contain the APGM form, while the iPGM is present in various parasites, including filariids. The siRNA treatment led to several phenotypic deformities in the intrauterine stages of treated female worms. A drastic reduction $(\sim 90 \%)$ was noticed in adult parasite motility along with a significant reduction $(80 \%)$ in the in vitro release of $\mathrm{mf}$ from female worms. Almost half the in vitro-treated L3 displayed sluggish movement. The important aspect of the study was the observation of in vivo survival and development of the siRNAtreated L3 in jirds that showed $\sim 45 \%$ reduction in the adult worm establishment. The information obtained from this study clearly indicates the necessity of $\mathrm{Bm}$ iPGM in the life cycle of $B$. malayi, and emphasizes its major role in female worm embryogenesis. The study validates Bm-iPGM as a potent anti-filarial drug target that can be utilized to design its novel inhibitors. Further structural and docking studies are underway to facilitate the design and synthesis of such Bm-iPGM inhibitors that would facilitate anti-filarial drug discovery programs.

\section{Additional file}

Additional file 1: Multilingual abstracts in the six official working languages of the United Nations.

\section{Competing interests}

The authors declare that they have no competing interests.

\section{Authors' contributions}

SMB conceived the study, analyzed the data, and drafted the manuscript. PKS and SK initiated the study, performed all the molecular and RNAi experiments, analyzed the data, and drafted the manuscript. MS and MP contributed to the experimentation. All the authors contributed to the writing of the manuscript and approved the submitted version.

\section{Acknowledgements}

The authors acknowledge the Council of Scientific and Industrial Research (CSIR), the University Grants Commission (UGC), and the Indian Council for Medical Research, New Delhi, India, for financial assistance in the form of Senior Research Fellowships to PKS, SK, MS, and MP. The financial assistance in the form of CSIR Network Project SplenDID is also gratefully acknowledged. We thank Mr. A. K. Roy and Mr. R. N. Lal for their excellent technical assistance in the maintenance of the $B$. malayi infection in the laboratory. This article bears CSIR-CDRI communication number 8421.

Received: 2 December 2012 Accepted: 21 March 2013

Published: 25 March 2013

\section{References}

1. WHO: Weekly epidemiological record. World Health Organisation 2011, 35:377-388

2. WHO: Lymphatic filariasis: reasons for hope. Geneva: World Health Organization; 1997:1-20.

3. WHO: Lymphatic filariasis elimination. Report of a meeting of the principles for the further enhancement of the public/private partnership. Amsterdam, The Netherlands; 1999:1-14.

4. Ismail MM, Jayakody RL, Weil GJ, Nirmalan N, Jayasinghe KS, Abeyewickrema W, Rezvi-Sheriff MH, Rajaratnam HN, Amarasekera N, de Silva DC, Michalski ML, Dissanaike AS: Efficacy of single dose combinations of albendazole, ivermectin and diethylcarbamazine for the treatment of bancroftian filariasis. Trans R Soc Trop Med Hyg 1998, 92:94-97.

5. Sharma DC: New goals set for filariasis elimination in India. Lancet Infect Dis 2002, 2:389. 
6. Ghedin E, Wang S, Spiro D, Caler E, Zhao Q, Crabtree J, Allen JE, Delcher AL, Guiliano DB, Miranda-Saavedra D, Angiuoli SV, Creasy T, Amedeo P, Haas B, El-Sayed NM, Wortman JR, Feldblyum T, Tallon L, Schatz M, Shumway M, Koo H, Salzberg SL, Schobel S, Pertea M, Pop M, White O, Barton GJ, Carlow CK, Crawford MJ, Daub J, Dimmic MW, Estes CF, Foster JM, Ganatra M, Gregory WF, Johnson NM, Jin J, Komuniecki R, Korf I, Kumar S, Laney S, Li BW, Li W, Lindblom TH, Lustigman S, Ma D, Maina CV, Martin DM, McCarter JP, McReynolds L, Mitreva M, Nutman TB, Parkinson J, Peregrin-Alvarez JM, Poole C, Ren Q, Saunders L, Sluder AE, Smith K, Stanke M, Unnasch TR, Ware J, Wei AD, Weil G, Williams DJ, Zhang Y, Williams SA, Fraser-Liggett C, Slatko B, Blaxter ML, Scott AL: Draft genome of the filarial nematode parasite Brugia malayi. Science 2007, 317:1756-1760.

7. Scott AL, Ghedin E, Nutman TB, McReynolds LA, Poole CB, Slatko BE, Foster JM: Filarial and Wolbachia genomics. Parasite Immunol 2012, 34:121-129.

8. Viney ME, Thompson FJ: Two hypotheses to explain why RNA interference does not work in animal parasitic nematodes. Int J Parasitol 2008, 38:43-47.

9. Kumar S, Chaudhary K, Foster JM, Novelli JF, Zhang Y, Wang S, Spiro D, Ghedin E, Carlow CKS: Mining predicted essential genes of Brugia malayi for nematode drug targets. PLOS One 2007, 2(11):e1189.

10. Jedrzejas MJ: Structure, function, and evolution of phosphoglycerate mutases: comparison with fructose-2,6-bisphosphatase, acid phosphatase, and alkaline phosphatase. Prog Biophys Mol Biol 2000, 73:263-287.

11. Zhang Y, Foster JM, Kumar S, Fougere M, Carlow CK: Cofactor-independent phosphoglycerate mutase has an essential role in Caenorhabditis elegans and is conserved in parasitic nematodes. J Biol Chem 2004, 279:37185-37190.

12. Landmann F, Foster JM, Slatko BE, Sullivan W: Efficient in vitro RNA interference and immunofluorescence-based phenotype analysis in a human parasitic nematode, Brugia malayi. Parasit Vectors 2012, 5:16.

13. Singh U, Misra S, Murthy PK, Katiyar JC, Agrawal A, Sircar AR: Immunoreactive molecules of Brugia malayi and their diagnostic potential. Serodiag Immun Inf D 1997, 8:207-212.

14. Mukherjee M, Misra S, Chatterjee RK: Optimization of test conditions for development of MTT as in vitro screen. Indian J Exp Biol 1997, 35:73-76.

15. Kushwaha S, Singh PK, Rana AK, Misra-Bhattacharya S: Cloning, expression, purification and kinetics of trehalose-6-phosphate phosphatase of filarial parasite Brugia malayi. Acta Trop 2011, 119:151-159.

16. Misra-Bhattacharya S, Katiyar D, Bajpai P, Tripathi RP, Saxena JK: 4-Methyl-7(tetradecanoyl)-2H-1-benzopyran-2-one a novel DNA topoisomerase II inhibitor with macrofilaricidal and embryostatic activity against subperiodic Brugia malayi. Parasitol Res 2004, 92:177-182.

17. Fraser AG, Kamath RS, Zipperlen P, Martinez-Campos M, Sohrmann M, Ahringer J: Functional genomic analysis of $C$. elegans chromosome I by systematic RNA interference. Nature 2000, 408:325-330.

18. Gonczy P, Echeverri C, Oegema K, Coulson A, Jones SJ, Copley RR, Duperon J, Oegema J, Brehm M, Cassin E, Hannak E, Kirkham M, Pichler S, Flohrs K, Goessen A, Leidel S, Alleaume AM, Martin C, Ozlu N, Bork P, Hyman AA: Functional genomic analysis of cell division in $\mathrm{C}$. elegans using RNAi of genes on chromosome III. Nature 2000, 408:331-336.

19. Piano F, Schetter AJ, Mangone M, Stein L, Kemphues KJ: RNAi analysis of genes expressed in the ovary of Caenorhabditis elegans. Curr Biol 2000, 10:1619-1622

20. Maeda I, Kohara Y, Yamamoto M, Sugimoto A: Large-scale analysis of gene function in Caenorhabditis elegans by high-throughput RNAi. Curr Biol 2001, 11:171-176.

21. Chander M, Setlow P, Lamani E, Jedrzejas MJ: Structural studies on a 2 3-diphosphoglycerate independent phosphoglycerate mutase from Bacillus stearothermophilus. J Struct Biol 1999, 126:156-165.

22. Jedrzejas MJ, Chander M, Setlow P, Krishnasamy G: Mechanism of catalysis of the cofactor-independent phosphoglycerate mutase from Bacillus stearothermophilus. Crystal structure of the complex with 2-phosphoglycerate. J Biol Chem 2000, 275:23146-23153.

23. Chevalier N, Rigden DJ, Van-Roy J, Opperdoes FR, Michels PA: Trypanosoma brucei contains a 2, 3-bisphosphoglycerate independent phosphoglycerate mutase. Eur J Biochem 2000, 267:1464-1472.

24. Guerra DG, Vertommen D, Fothergill-Gilmore LA, Opperdoes FR, Michels PA: Characterization of the cofactor-independent phosphoglyceratemutase from Leishmania Mexicana mexicana. Histidines that coordinate the two metal ions in the active site show different susceptibilities to irreversible chemical modification. Eur J Biochem 2004, 271:1798-1810.

25. Besteiro S, Barrett MP, Riviere L, Bringaud F: Energy generation in insect stages of Trypanosoma brucei: metabolism in flux. Trends Parasitol 2005, 21:185-191.

26. Djikeng A, Raverdy S, Foster J, Bartholomeu D, Zhang Y, El-Sayed NM, Carlow C: Cofactor-independent phosphoglycerate mutase is an essential gene in procyclic form Trypanosoma brucei. Parasitol Res 2007, 100:887-892.

27. Foster JM, Davis PJ, Raverdy S, Sibley MH, Raleigh EA, Kumar S, Carlow CKS: Evolution of Bacterial Phosphoglycerate Mutases: Non-Homologous Isofunctional Enzymes Undergoing Gene Losses, Gains and Lateral Transfers. PLoS One 2010, 5(10):e13576.

28. Guo S, Kemphues $\mathrm{KJ}$ : par-1, a gene required for establishing polarity in $C$. elegans embryos, encodes a putative Ser/Thr kinase that is asymmetrically distributed. Cell 1995, 81:611-620.

29. Fire A, Xu S, Montgomery MK, Kostas SA, Driver SE, Mello CC: Potent and specific genetic interference by double-stranded RNA in Caenorhabditis elegans. Nature 1998, 391:806-811.

30. Hussein AS, Kichenin K, Selkirk ME: Suppression of secreted acetylcholinesterase expression in Nippostrongylus brasiliensis by RNA interference. Mol Biochem Parasitol 2002, 122:91-94.

31. Geldhof P, Murray L, Couthier A, Gilleard JS, MCLauchlan G, Knox DP, Britton C: Testing the efficacy of RNA interference in Haemonchus contortus. Int J Parasitol 2006, 36:801-810.

32. Kotze AC, Bagnall NH: RNA interference in Haemonchus contortus: suppression of beta-tubulin gene expression in L3, L4 and adult worms in vitro. Mol Biochem Parasitol 2006, 145:101-110.

33. Samarasinghe $B$, Knox DP, Britton C: Factors affecting susceptibility to RNA interference in Haemonchus contortus and in vivo silencing of an $\mathrm{H} 11$ aminopeptidase gene. Int J Parasitol 2011, 41:51-59.

34. Islam MK, Miyoshi T, Yamada M, Tsuji N: Pyrophosphatase of the roundworm Ascaris suum plays an essential role in the worm's molting and development. Infect Immun 2005, 73:1995-2004.

35. Issa Z, Grant WN, Stasiuk S, Shoemaker CB: Development of methods for RNA interference in the sheep gastrointestinal parasite, Trichostrongylus colubriformis. Int J Parasitol 2005, 35:935-940.

36. Chen $N$, Xu MJ, Nisbet AJ, Huang CQ, Lin RQ, Yuan ZG, Song HQ, Zhu XQ: Ascaris suum: RNAi mediated silencing of enolase gene expression in infective larvae. Exp Parasitol 2011, 127:142-146.

37. Visser A, Geldhof P, de Maere V, Knox DP, Vercruysse J, Claerebout E: Efficacy and specificity of RNA interference in larval life-stages of Ostertagia ostertagi. Parasitology 2006, 133:777-783.

38. Lustigman S, Zhang J, Liu J, Oksov Y, Hashmi S: RNA interference targeting cathepsin $L$ and Z-like cysteine proteases of Onchocerca volvulus confirmed their essential function during L3 molting. Mol Biochem Parasitol 2004, 2004(138):165-170.

39. Ford L, Guiliano DB, Oksov Y, Debnath AK, Liu J, Williams SA, Blaxter ML, Lustigman S: Characterization of a novel filarial serine protease inhibitor Ov-SPI-1, from Onchocerca volvulus, with potential multifunctional roles during development of the parasite. J Biol Chem 2005, 280:40845-40856.

40. Aboobaker AA, Blaxter ML: Use of RNA interference to investigate gene function in the human filarial nematode parasite Brugia malayi. Mol Biochem Parasitol 2003, 129:41-51.

41. Song C, Gallup JM, Day TA, Bartholomay LC, Kimber MJ: Development of an in vivo RNAi protocol to investigate gene function in the filarial nematode, Brugia malayi. PLoS Pathog 2010, 6:e1001239.

42. Singh M, Singh PK, Misra-Bhattacharya S: RNAi mediated silencing of ATPase RNA helicase gene in adult filarial parasite Brugia malayi impairs in vitro microfilaria release and adult parasite viability. J Biotechnol 2012, 157:351-358.

43. Elbashir SM, Lendeckel W, Tuschl T: RNA interference is mediated by 21and 22-nucleotide RNAs. Genes Dev 2001, 15:188-200.

44. Elbashir SM, Martinez J, Patkaniowska A, Lendeckel W, Tuschl T: Functional anatomy of siRNAs for mediating efficient RNAi in Drosophila melanogaster embryo lysate. EMBO J 2001, 20:6877-6888.

45. Elbashir SM, Harborth J, Weber K, Tuschl T: Analysis of gene function in somatic mammalian cells using small interfering RNAs. Methods 2002, 26:199-213.

46. Holen T: Mechanisms of RNAi: mRNA cleavage fragments may indicate stalled RISC. J RNAi Gene Silencing 2005, 1:21-25. 
47. Pfarr $\mathrm{K}$, Heider $\mathrm{U}$, Hoerauf $\mathrm{A}$ : RNAi mediated silencing of actin expression in adult Litomosoides sigmodontis is specific, persistent and results in a phenotype. Int J Parasitol 2006, 36:661-669.

48. Reynolds A, Anderson EM, Fedorov AY, Robinson K, Leake D, Karpilow J, Marshall WS, Khvorova A: Induction of the interferon response by siRNA is cell type- and duplex length-dependent. RNA 2006, 12(6):988-993.

49. Bantounas I, Phylactou LA, Uney JB: RNA interference and the use of small interfering RNA to study gene function in mammalian systems. J Mol Endocrinol 2004, 33:545-557.

50. Kushwaha S, Singh PK, Shahab M, Pathak M, Bhattacharya SM: In Vitro Silencing of Brugia malayi Trehalose-6-Phosphate Phosphatase Impairs Embryogenesis and In Vivo Development of Infective Larvae in Jirds. PLoS Negl Trop Dis 2012, 6(8):e1770.

51. Tsang WY, Lemire BD: Mitochondrial genome content is regulated during nematode development. Biochem Biophys Res Commun 2002, 291:8-16.

52. Li BW, Rush AC, Jiang DJ, Mitreva M, Abubucker S, Weil GJ: Genderassociated genes in filarial nematodes Are important for reproduction and potential intervention targets. PLoS Negl Trop Dis 2011, 5(1):e947.

53. Westram A, Lloyd JR, Roessner U, Riesmeier JW, Kossmann J: Increases of 3-phosphoglyceric acid in potato plants through antisense reduction of cytoplasmic phosphoglycerate mutase impairs photosynthesis and growth, but does not increase starch contents. Plant Cell Environ 2002, 25:1133-1143.

54. Zhao Z, Assmann SM: The glycolytic enzyme, phosphoglycerate mutase, has critical roles in stomatal movement, vegetative growth, and pollen production in Arabidopsis thaliana. J Exp Bot 2011, 62:5179-5189.

55. Albert MA, Haanstra JR, Hannaert V, Van-Roy J, Opperdoes FR, Bakker BM, Michels PA: Experimental and in silico analyses of glycolytic flux control in bloodstream form Trypanosoma brucei. J Biol Chem 2005, 280:28306-28315.

56. Leyva-Vazquez MA, Setlow P: Cloning and nucleotide sequences of the genes encoding triose phosphate isomerase, phosphoglycerate mutase, and enolase from Bacillus subtilis. J Bacteriol 1994, 176:3903-3910.

57. Morris VL, Jackson DP, Grattan M, Ainsworth T, Cuppels DA: Isolation and sequence analysis of the Pseudomonas syringae pv. tomato gene encoding a 2,3-diphosphoglycerate-independent phosphoglyceromutase. J Bacteriol 1995, 177:1727-1733.

doi:10.1186/2049-9957-2-5

Cite this article as: Singh et al:: In vitro gene silencing of independent phosphoglycerate mutase (iPGM) in the filarial parasite Brugia malayi. Infectious Diseases of poverty 2013 2:5.

\section{Submit your next manuscript to BioMed Central and take full advantage of:}

- Convenient online submission

- Thorough peer review

- No space constraints or color figure charges

- Immediate publication on acceptance

- Inclusion in PubMed, CAS, Scopus and Google Scholar

- Research which is freely available for redistribution 